\title{
SEM-EDX and SEM-CL to Characterize Lapis Lazuli from Different Provenances
}

\author{
A. Re, ${ }^{*}$ A. Lo Giudice, ${ }^{*}$ D. Angelici, ${ }^{*}$ G. Pratesi** \\ * Dipartimento di Fisica Sperimentale, Università di Torino and INFN, Sezione di Torino, Via P. Giuria 1, \\ 10125, Torino, Italy \\ ** Dipartimento di Scienze della Terra and Museo di Storia Naturale, Università di Firenze, Via G. La Pira 4, \\ 50121 Firenze, Italy
}

Lapis lazuli has been used for more than 7000 years for the manufacture of precious objects and jewels. The main quarries for this stone are still active in Afghanistan, but there are other quarries that could have been exploited since antiquity in the Pamir mountains (Tajikistan), in Pakistan and in Siberia. For this reason a provenance study of lapis lazuli could provide answers to some important issues, in particular the use and the dissemination of this rock through historic commercial routes. In the present work a systematic study has been performed on lapis lazuli from different quarries using Energy Dispersive X-ray spectroscopy (EDX) and cathodoluminescence (CL). The aim of this characterization is to identify the main phases present in the stone and to find some markers to distinguish among provenances.

Lapis lazuli samples that have been analyzed come from the Mineralogical Museum of Florence and they have been collected in four sources: Sar-e-Sang Badakhshan (Afghanistan), Ovalle (Chile), Irkutsk near Lake Baikal (Siberia) and Pamir mountains (Tajikistan) [1]. Fifteen thin sections have been prepared and a preliminary characterization of the samples has been carried out by means of optical microscope and cold-CL (CL8200 Mk3). After coating, selected grains have been studied by means of SEM-EDX (Cambridge Stereoscan S360 with an Oxford PentaFET EDX), using also a set of standards for the quantitative analysis, and SEM-CL (Oxford MonoCL).

$\mathrm{CL}$ is a powerful technique to rapidly identify the mineral phase distribution. In some cases this technique is able to reach a sensitivity of some ppm, because of the presence of a particular element that activates the luminescence, or if it is present in a very small quantity. Unfortunately it is normally not easy to quantify the element contents due to a competition between luminescence centers (i.e. the intensity of CL signal) and chemical elements that work as quenchers [2]. In any case by means of SEM-EDX and SEM-CL it is possible to obtain both the elemental composition of a small area (few $\mu \mathrm{m}$ square) and its luminescence response in the UV-Visible spectral range.

Due to the rocks heterogeneity, it is very difficult to identify provenance markers by analyzing elemental composition of the whole rocks; hence investigation has been focused on mineral phases. SEM-CL spectra have been collected from all the main luminescent phases, together with SEM-EDX spectra of the same crystals, used for the identification of the minerals.

Despite the limited number of analyzed samples, results are promising and the experimental differences among rocks from different sources are significant. The data have already been integrated by Ion Beam Analyses measurements, confirming the results obtained with the electron probe [1][3].

The principal phase of lapis lazuli is lazurite, a blue mineral that doesn't show luminescence. One of the main accessory minerals in all the Asian samples is diopside, while in Chilean samples wollastonite is present. These two phases have different luminescence spectra and this fact makes Chilean samples very easy to be distinguished from others [1][4]. Lapis Lazuli from Pamir is characterized by the presence of a cancrinite phase with a strong UV emission and a very peculiar spectrum [1]. Other phases that have been recognized in 
samples from all the provenances are the calcite and the K-feldspar. Both of these phases doesn't present differences among provenances, neither for the main elements detected by means of EDX, nor for the luminescence spectra collected by means of CL. The only difference among spectra is the intensity of the luminescence bands, but it's related to the crystal and not to the provenance. In Figure 1 the K-feldspar spectra are presented: this phase shows a light-blue emission in CL and its spectrum is characterized by a broad band centered at $455 \mathrm{~nm}$, due to Al-O-Al defects [3]. Calcite presents a vivid orange color in cathodoluminescence and its spectra are shown in Figure 1: they are characterized by the presence of an intense band at $625 \mathrm{~nm}$, due to $\mathrm{Mn}^{2+}$ substituting $\mathrm{Ca}^{+}$, and by a weaker band at $350 \mathrm{~nm}$, due to intrinsic luminescence [3].

The characterization presented in this work is very useful to better know the rock lapis lazuli and is propaedeutic for a successive work devoted to the analysis of works of art. In fact there are some limitations in using electron microscopy for the characterization of art-objects, because it's impossible to put them in vacuum and often they are too big for a SEM chamber. The markers obtained with the electron gun are also visible with Ion Beam Analysis and these techniques have been proven to be applicable on lapis lazuli rocks and works of art having various shapes [4]. These techniques are very suitable to the Cultural Heritage field, because they are non-invasive and can be utilized in air without any sample pre-treatment, obtaining simultaneously major and trace element.

References

[1] A. Lo Giudice et al., Anal. Bioanal. Chem. 395 (2009) 2211

[2] M. Pagel et al., Cathodoluminescence in geosciences, Springer, Berlin, 2000

[3] A. Re et al., Nucl. Instr. and Meth. B (2011) in press

[4] S. Calusi et al, Nucl. Instr. and Meth. B 266 (2008) 2306

[5] This work is funded by National Institute of Nuclear Physics, INFN (FARE and DANTE experiments). The authors gratefully acknowledge the "Compagnia di San Paolo" for the funding of a Ph.D. scholarship on Physics applied to Cultural Heritage.
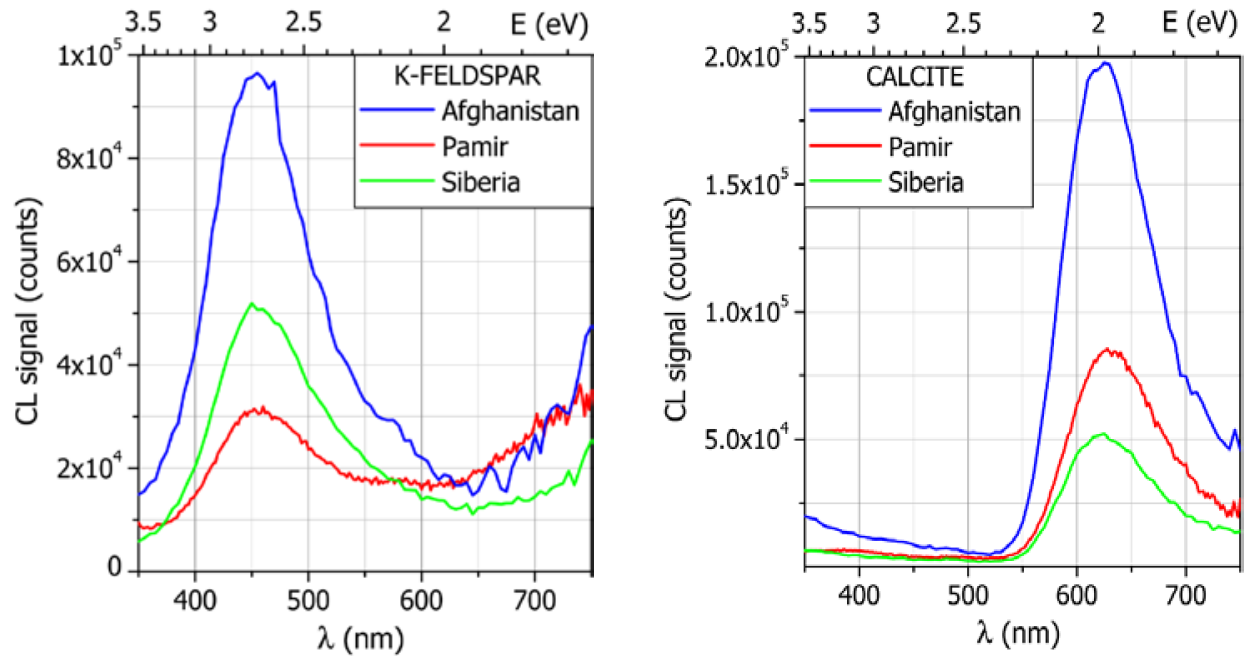

FIG. 1. CL spectra of: K-feldspar and calcite from lapis lazuli samples coming from different provenances; the spectra are already corrected for the response of the instrument. 